Supporting Information for

\title{
LARGE-SCALE SINGLE-CELL TRAPPING AND IMAGING USING MICROWELL ARRAYS
}

\author{
Jacqueline R. Rettig \& Albert Folch* \\ Department of Bioengineering, Box 352255, \\ University of Washington, Seattle, WA 98195-2255 \\ afolch@u.washington.edu
}

\section{CONTENTS}

VIABILITY OF PROCEDURE .................................... $\quad$ S-2

Figure S-1. 3T3 cells labeled with calcein AM and ethidium homodimer-1........ S-3

Figure S-2. RBL-1 cells labeled with calcein AM and ethidium homodimer-1.......S-5

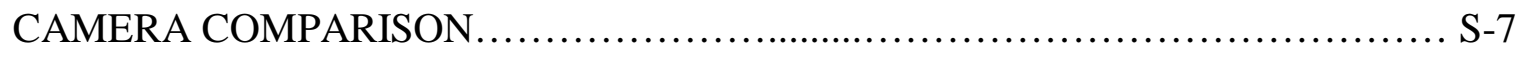

Table S-1. Camera comparison ( $4 \times$ objective) ................................. S-9

Figure S-3. Camera considerations........................................ S-10 


\section{VIABILITY OF PROCEDURE}

For viability imaging, $2 \mu \mathrm{M}$ calcein $\mathrm{AM}$ and $4 \mu \mathrm{M}$ ethidium homodimer-1 (EthD-1, Molecular Probes) were added to the cells as they settled into the microwells for $30-40 \mathrm{~min}$. EthD-1 is a red-fluorescent cell-impermeant dead-cell dye, which undergoes a $\sim 40$-fold fluorescence enhancement upon binding to nucleic acids, hence it is commonly used as a reporter of cell death.

For 3 T3 fibroblasts (Fig. S-1), PDMS microwell arrays ( $D=25 \mu \mathrm{m}, H=23 \mu \mathrm{m}, C=35 \mu \mathrm{m})$ were coated with $1 \mathrm{mg} / \mathrm{mL}$ BSA for $1 \mathrm{hr}$, rinsed with $\mathrm{dI} \mathrm{H}_{2} \mathrm{O}$, dried with a $\mathrm{CO}_{2}$ stream, and filled with serum-containing medium in $\mathrm{CO}_{2}$. $3 \mathrm{~T} 3$ fibroblasts were trypsinized, centrifuged at $800 \mathrm{rpm}$ for $5 \mathrm{~min}$, resuspended in serum-containing medium with $2 \mu \mathrm{M}$ calcein $\mathrm{AM}$ and $4 \mu \mathrm{M}$ EthD-1, seeded on microarrays at $4.3 \times 10^{3}$ cells $/ \mathrm{mm}^{2}$, and allowed to settle for $\sim 40 \mathrm{~min}$. The microarray was then rinsed with serum-free DMEM to remove excess cells and placed in the incubator for $15 \mathrm{~min}$ and then micrographs were taken (about 75-90 minutes after trypsinization of the cells). The fluorescence micrographs are shown separately in Fig. S-1a (calcein AM) and Fig. S-1b (EthD-1). The overlay of the two micrographs is shown in Fig. S-1c.

For RBL-1 cells (Fig. S-2), PDMS microwell arrays ( $D=25 \mu \mathrm{m}, H=23 \mu \mathrm{m}, C=35 \mu \mathrm{m})$ were filled with serum-containing medium in $\mathrm{CO}_{2}$. RBL-1 cells were centrifuged at $800 \mathrm{rpm}$ for $5 \mathrm{~min}$, resuspended in serum-free medium with $2 \mu \mathrm{M}$ calcein $\mathrm{AM}$ and $4 \mu \mathrm{M}$ EthD-1, seeded on microarrays at $2.7 \times 10^{3}$ cells $/ \mathrm{mm}^{2}$, and allowed to settle $30 \mathrm{~min}$. The microarrays were then rinsed with serum-free DMEM to remove excess cells and micrographs were taken. The fluorescence micrographs are shown separately in Fig. S-2a (calcein AM) and Fig. S-2b (EthD-1). The overlay of the two micrographs is shown in Fig. S$2 c$. 
Figure S-1. 3T3 cells labeled with calcein AM and ethidium homodimer-1 (EthD). (a) Fluorescence micrograph of 3T3 fibroblasts in microwells labeled with calcein AM; (b) corresponding micrograph of EthD; (c) color overlay of (a) and (b) (rotated $90^{\circ}$ and enlarged) with calcein AM shown green and ethidium homodimer-1 shown red. Over 12,500 microwells are shown.

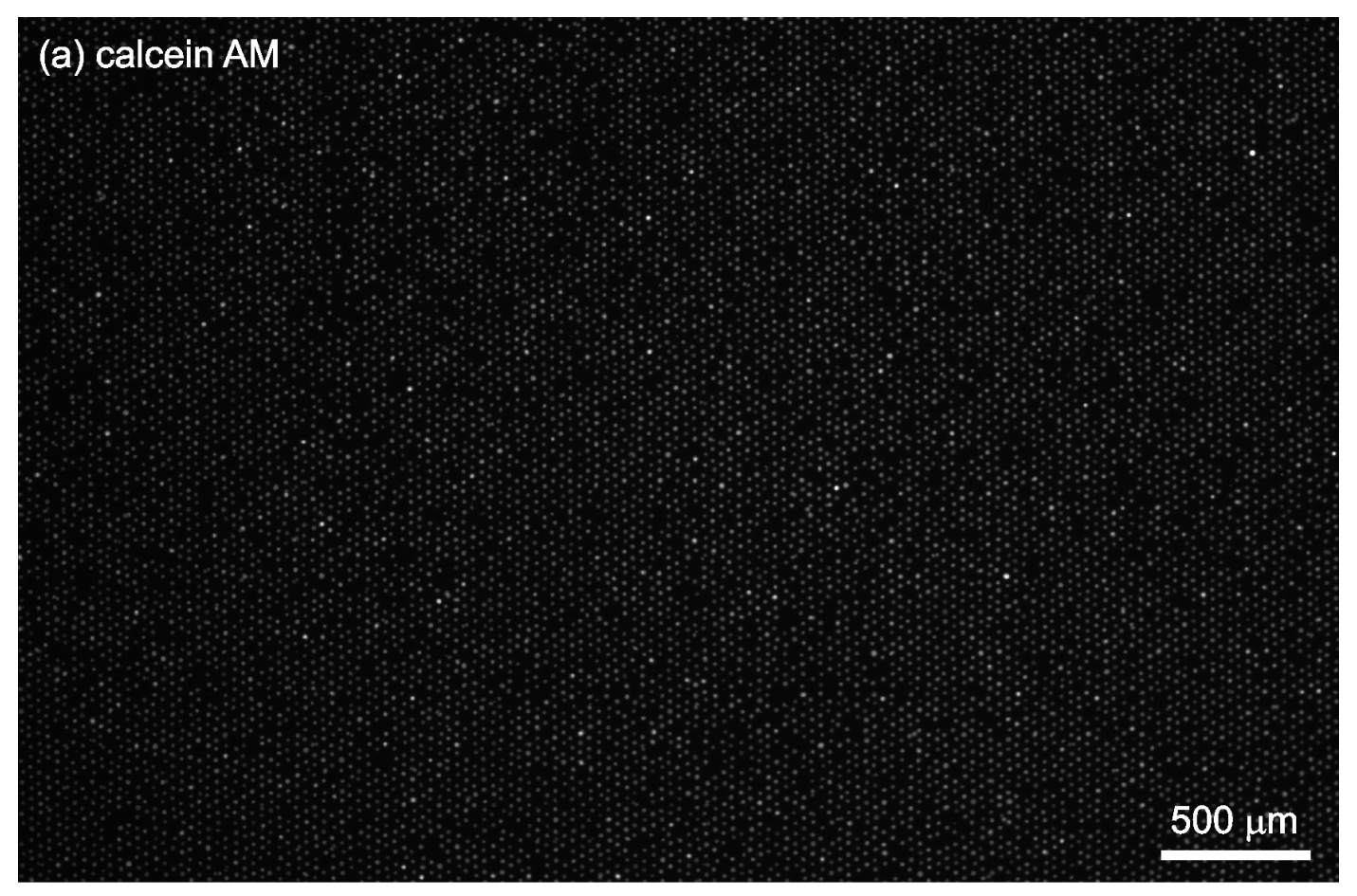

(b) ethidium homodimer-1 
(c) overlay of calcein AM and ethidium homodimer-1 
Figure S-2. RBL-1 cells labeled with calcein AM and ethidium homodimer-1 (EthD). (a)

Fluorescence micrograph of RBL-1 cells in microwells labeled with calcein AM; (b) corresponding micrograph of EthD labeling; (c) color overlay of (a) and (b) (rotated $90^{\circ}$ and enlarged) with calcein AM labeling shown in green and EthD labeling shown in red. Over 12,500 microwells are shown.

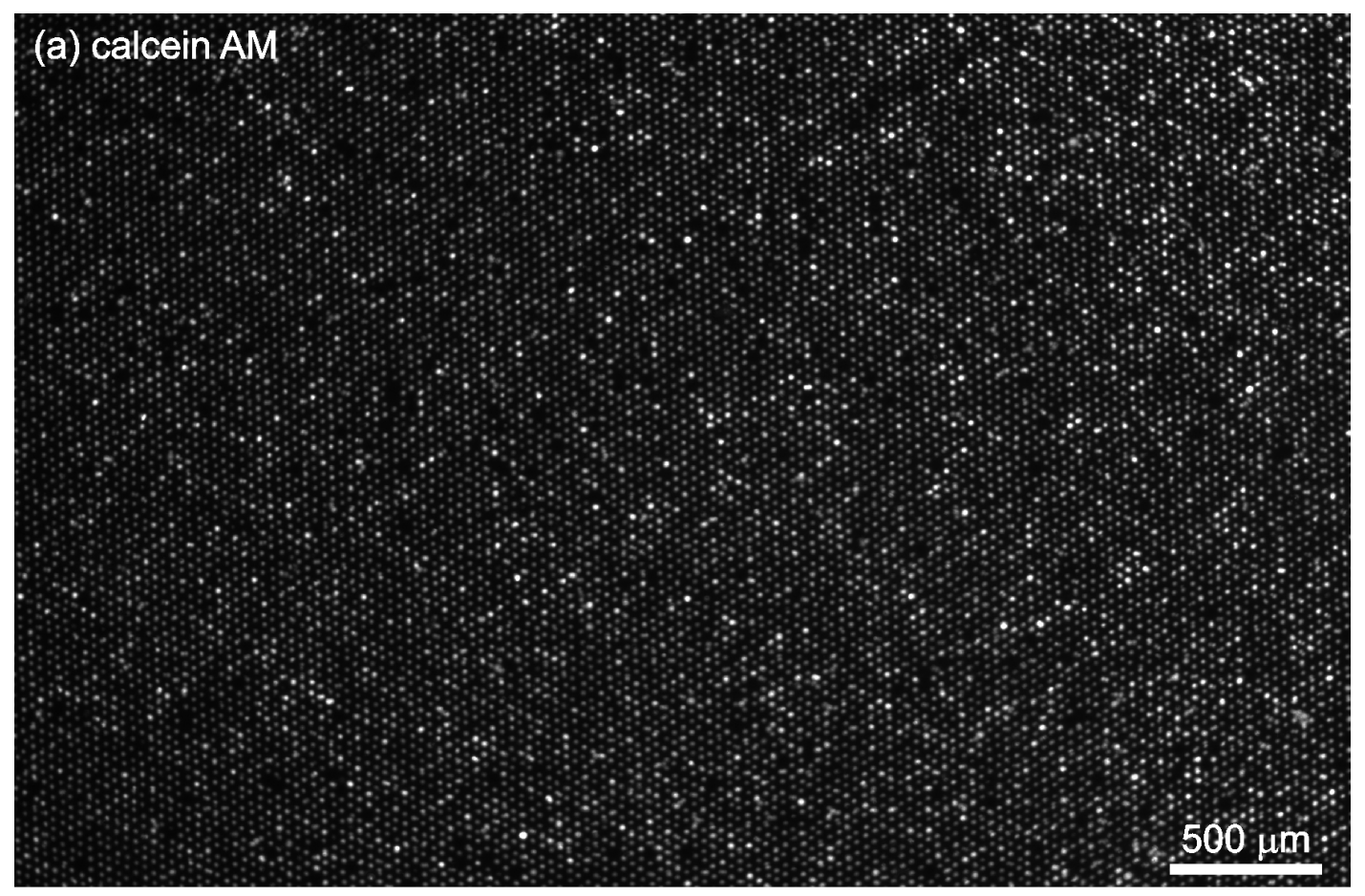

(b) ethidium homodimer-1 


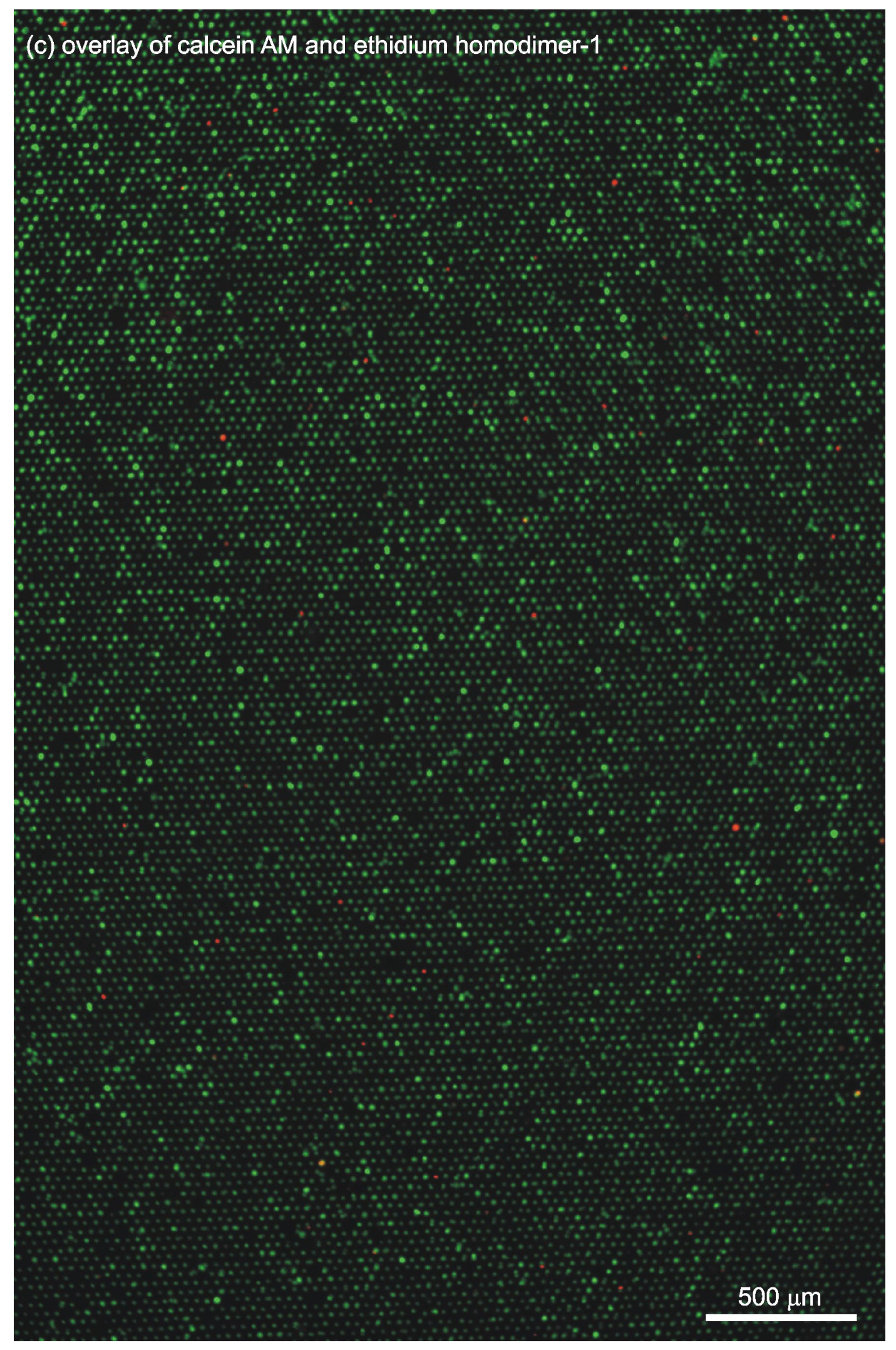




\section{CAMERA COMPARISON}

For large-area fluorescence microscopy imaging, we compared two different cameras simultaneously connected to two different ports of a Nikon Eclipse TE2000-U microscope using a $4 \times$ objective. The cameras include the Orca-AG (C4742-80-12AG) and the Orca-HR (C474295-12HR) (Hamamatsu Photonics, Hamamatsu City, Japan), and both are 12-bit, cooled-CCD cameras of similar sensitivity. Table S-1 shows a comparison of the capabilities of the two cameras with regards to imaging microwell arrays.

For the purposes of illustration, as summarized in Table S-1, we compared two high-end 12-bit CCD cameras from the same manufacturer: the Orca-AG (a high-sensitivity camera that is popularly used in many biological laboratories and that features a chip size of 1,344 pixels $\times$ 1,024 pixels) and the newer, large-chip Orca-HR (a camera with a slightly lower sensitivity that features a chip size of 4,000 pixels $\times 2,624$ pixels, the largest commercially-available CCD camera chip to the best of our knowledge). The Orca-HR chip features a pixel size (5.9 $\mu \mathrm{m} \times 5.9$ $\mu \mathrm{m})$ that is $\sim 16 \%$ smaller in area than that of the Orca-AG chip $(6.45 \mu \mathrm{m} \times 6.45 \mu \mathrm{m})$, resulting in a correspondingly lower pixelation at the same magnification.

Cropped phase and fluorescence images of $D=25 \mu \mathrm{m}, H=23 \mu \mathrm{m}$ microwells taken with the Orca-HR with a $4 \times$ objective are shown in Fig. S-3a and S-3b, respectively. The 3 T3 fibroblasts were labeled with calcein AM. As shown in Fig. S-3c, the Orca-HR CCD chip covers a field of view $\left(22.9 \mathrm{~mm}^{2}\right.$ at $\left.4 \times\right)$ that is more than 6 times larger than that of the Orca-AG $\left(3.6 \mathrm{~mm}^{2}\right.$ at $\left.4 \times\right)$, resulting in 6.4 times more microwells per picture. For example, the optimum microwell size for RBL-1 cells, $D=20 \mu \mathrm{m}$, enables visualization of over 29,000 microwells with the Orca-HR camera at $4 \times$ magnification if a $30 \mu \mathrm{m}$ center-to-center $(C)$ microwell spacing (well to well separation $S=C-D=10 \mu \mathrm{m}$ ) is used, which represents $\sim 26,400$ single cells at $90 \%$ single-cell occupancy; by comparison, the Orca-AG camera is capable of imaging only 4,100 single cells simultaneously using the same array and microwell occupancy (see Table S-1 for comparisons between the two cameras using other microwell spacings). 
We note that the larger imaging area of the Orca-HR comes at a loss in sensitivity: the readout noise (r.m.s.) of the Orca-AG is $\sim 46 \%$ of that of the Orca-HR (see Table S-1). Nevertheless, the lower sensitivity may be sufficient for many fluorescence imaging applications provided a sufficiently-bright dye is used. As a demonstration, the insets in Fig. S-3a and S-3b show phase and fluorescence images of a single $3 \mathrm{~T} 3$ fibroblast that occupies $~ 45 \%$ of the microwell pixels; the upper insets in Fig. S-3a and S-3b are cropped from a large $(5.9 \mathrm{~mm} \times 3.9 \mathrm{~mm})$ image of a microwell array (containing 21,000 microwells) taken with the Orca-HR and a $4 \times$ objective; for comparison the lower insets in Fig. S-3a and S-3b show the same cell in images taken with the Orca-AG and a $4 \times$ objective. At this magnification, pixelation starts to be apparent when looking at single cells, but a distinct, non-spherical morphology of the cells is discernible. The pixel intensities after background subtraction for the same pixels shown in the upper and lower insets of Fig. S-3b are shown in the graphs in Fig. S-3d and S-3e, respectively. These pixel intensity graphs clearly depict that the sensitivity of either camera is sufficient to detect cell fluorescence above the background noise at $4 \times$ magnification. From Fig. S-3 we conclude that the Orca-HR, despite its lower sensitivity, is very well suited for detecting cell viability fluorescence signals from large numbers of single cells. It is possible that for applications yielding much lower cell brightness, the sensitivity of the Orca-HR may not be sufficient. Nevertheless, we believe that the simultaneous detection of tens of thousands of single-cell responses represents a powerful strategy for identifying "rare" responses in a variety of bioanalytical assays. 
Table S-1. Camera comparison (4× objective). The number of microwells per $4 \times$ field of view and the number of pixels per microwell are shown for two cameras (Hamamatsu Orca-HR and Orca-AG) and a range of center-to-center spacings $(C=25-55 \mu \mathrm{m})$. The well-to-well spacing is assumed to be $S=10 \mu \mathrm{m}$. The area of the $4 \times$ field of view, pixel size, and readout noise of each camera is also shown. Compared to the Orca-AG, at any given $C$ the Orca-HR can image 6.4 times more microwells with a resolution $\sim 20 \%$ higher but has more than twice the readout noise (lower sensitivity).

\begin{tabular}{|c|c|c|c|}
\hline \multirow{2}{*}{$\begin{array}{l}\text { Microwell } \\
\text { Center-to- } \\
\text { Center } \\
\text { Spacing }(C) \\
(\mu \mathrm{m})^{\star \star *}\end{array}$} & \multicolumn{3}{|c|}{$\begin{array}{l}\text { \# of Microwells* } \\
\qquad \text { @ \# Pixels/Microwell }\end{array}$} \\
\hline & Orca-AG & Orca-HR & $\begin{array}{c}\text { Ratio } \\
\text { (HR:AG) }\end{array}$ \\
\hline 25 & @ 670 & $\begin{array}{r}42,250 \\
\quad @ 80\end{array}$ & \multirow{7}{*}{$6.4 @ 1.20$} \\
\hline 30 & $\begin{array}{l}4,600 \\
\quad 120\end{array}$ & $\begin{array}{r}29,350 \\
\text { @ } 140\end{array}$ & \\
\hline 35 & $\begin{array}{l}3,350 \quad \text { @ } 190 \\
\quad 190\end{array}$ & $\begin{array}{r}21,550 \\
\quad @ 230\end{array}$ & \\
\hline 40 & $\begin{array}{l}2,600 \\
\quad @ 270\end{array}$ & $\begin{array}{r}16,500 \\
@ 330\end{array}$ & \\
\hline 45 & $\begin{array}{l}2,050 \\
\quad @ 370\end{array}$ & $\begin{array}{r}13,050 \\
\quad \text { @ } 440\end{array}$ & \\
\hline 50 & $\begin{array}{l}1,650 \\
\quad \text { @ } 480\end{array}$ & $\begin{array}{r}10,550 \\
@ 580\end{array}$ & \\
\hline 55 & $\begin{array}{l}1,350 \\
\quad 610\end{array}$ & $\begin{array}{l}8,750 \\
\quad \text { @ } 730\end{array}$ & \\
\hline $\begin{array}{l}\text { Imaging } \\
\text { area }\left(\mathrm{mm}^{2}\right)\end{array}$ & 3.6 & 22.9 & 6.4 \\
\hline $\begin{array}{l}\text { On-chip } \\
\text { pixel size } \\
(\mu \mathrm{m} \times \mu \mathrm{m})\end{array}$ & $6.45 \times 6.45$ & $5.9 \times 5.9$ & $0.91 \times 0.91$ \\
\hline $\begin{array}{l}\text { Readout } \\
\text { noise } \\
\text { (r.m.s.) }\end{array}$ & 6 electrons & 13 electrons & 2.17 \\
\hline
\end{tabular}

* Rounded to the nearest multiple of 50

** Rounded to the nearest multiple of 10

*** Hexagonal array 
Figure S-3. Camera considerations. Phase-contrast (a) and fluorescence (b) images of calcein AM-labeled 3T3 fibroblasts in a PDMS array of $25 \mu \mathrm{m}$ diam. microwells spaced $35 \mu \mathrm{m}$ centerto-center taken with a Hamamatsu Orca-HR camera and a $4 \times$ objective. Upper and lower insets in (a) and (b) show an enlargement of a single cell cropped from the larger image obtained using a 4x objective with the Orca-HR and the Orca-AG, respectively. Note the non-round shape of some cells as they spread and attach on the side walls of the microwells. (c) Relative sizes of the field of view of the large-chip Orca-HR (black rectangle) and the high-sensitivity Orca-AG (white rectangle) cameras, with the gray square representing the portion of the field of view shown in (a) and (b). Note that the total area of the field of view of the Orca-HR is more than $6 \times$ larger than that of the Orca-AG. (d and e) 3-d graphs of the pixel intensities of the same cell shown in the insets in (b) for both the Orca-HR (d) and the Orca-AG (e).
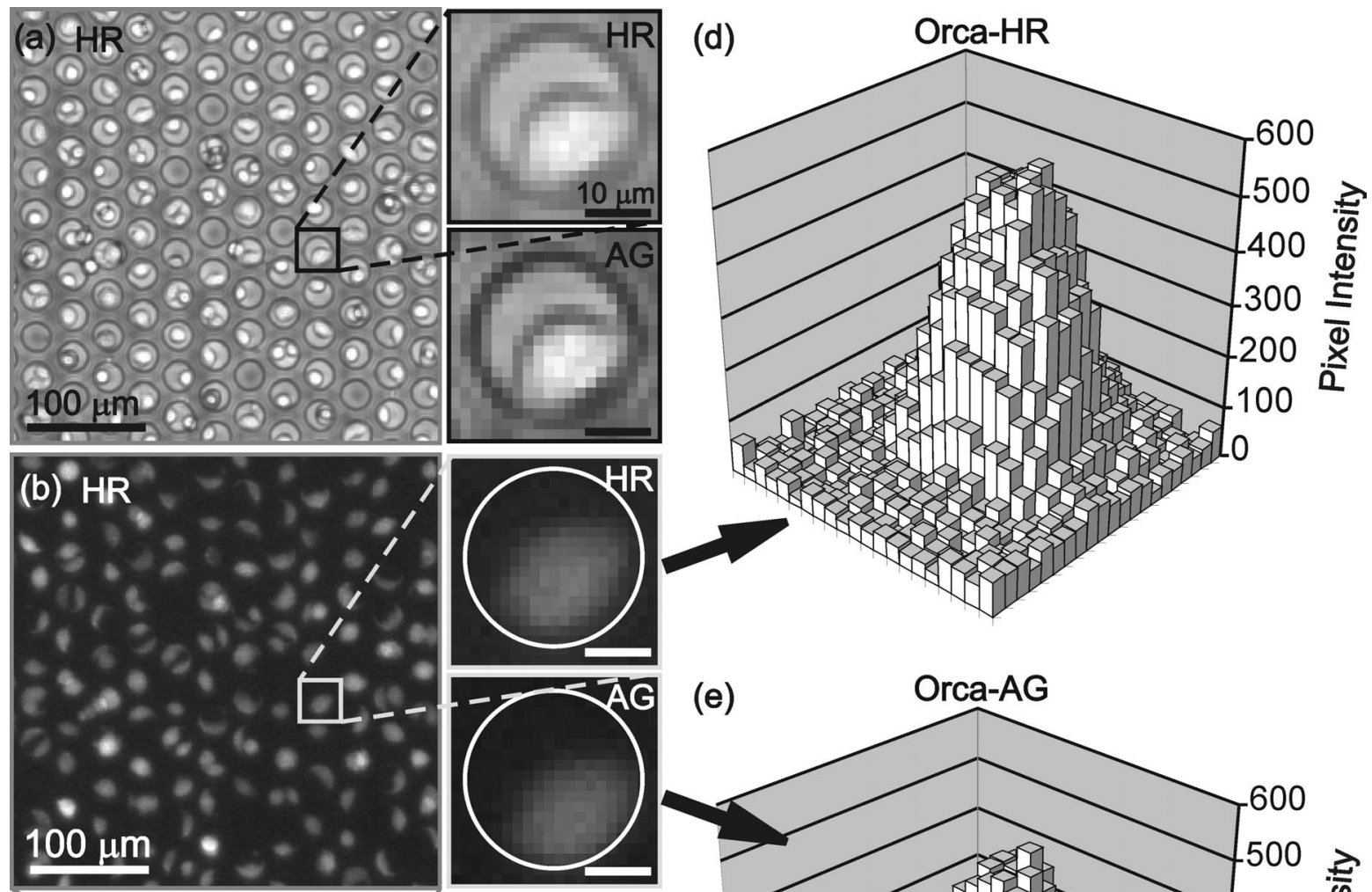

(d)

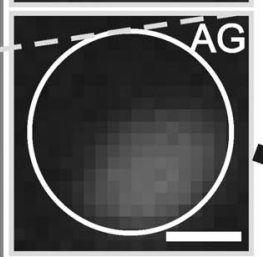

(c)
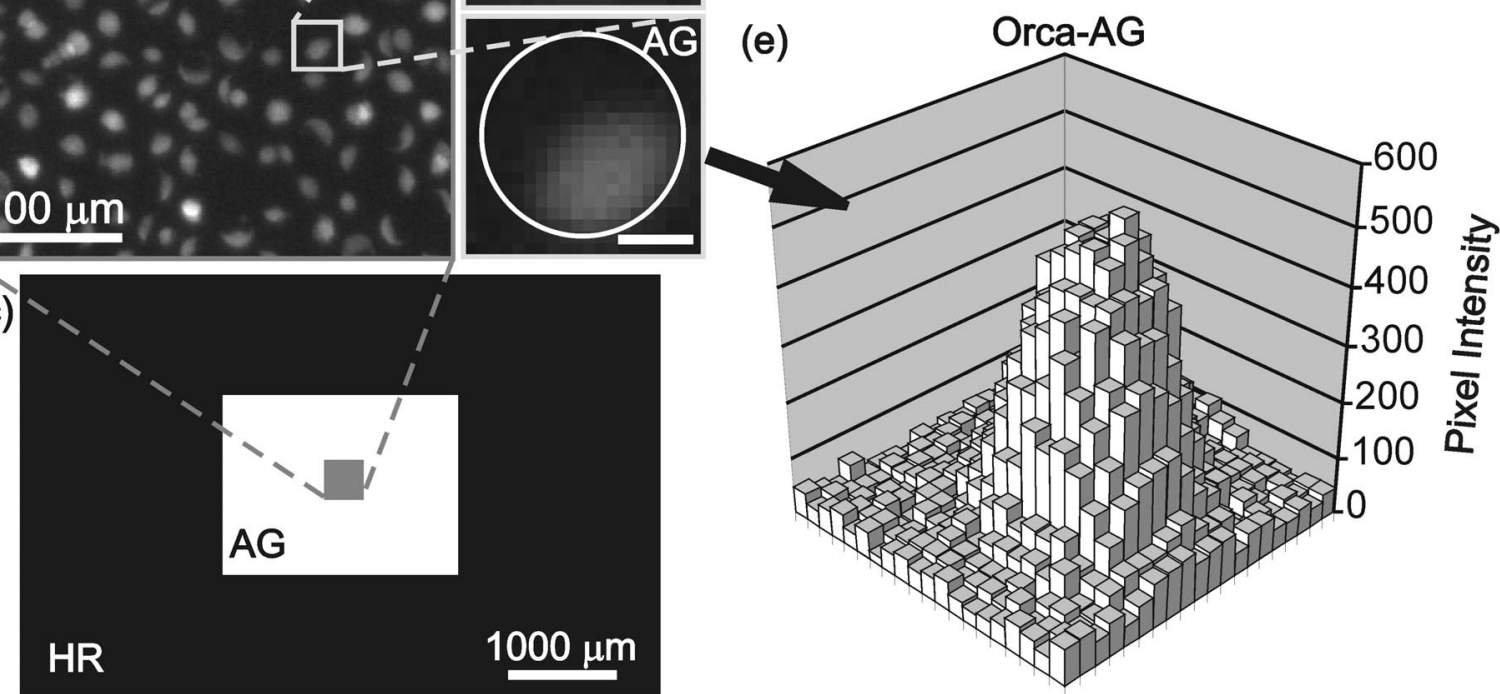